\title{
Vaginal entrapment of bathwater: a source of extra-urethral incontinence
}

\author{
Karen Psooy, MD, FRCSC; ; Jason P. Archambault, MD
}

\begin{abstract}
This report presents 2 cases of females (a prepubertal and a premenopausal female) with dribbling "incontinence" caused by vaginal entrapment of bathwater. In the first case, an 8-year-old girl presented with a history of bothersome wetness of her underwear, noted mainly in the morning. History revealed that she bathed almost nightly. She was instructed to shower or bath only, each for one week. The wetting occurred only after bathing. In the second case, a 39-year-old female, G2P2 with normal genitalia noted occasional, yet significant, dribbling into her underwear only after baths. She concluded this to be bathwater accumulating in her vagina, which she evacuates digitally. The differential diagnosis of dribbling incontinence in females of any age should include the extra-urethral cause of entrapped fluids of immersion. Although this condition may be rare, it is simple to rule out on history based on its temporal association with fluid immersion. Once diagnosed, the management is behavioural.
\end{abstract}

Can Urol Assoc J 2010;4(5):E123-126

\section{Introduction}

The International Continence Society (ICS) acknowledges that urinary leakage may need to be distinguished from a vaginal discharge. ${ }^{1}$ However, consideration of the discharge being a non-physiological fluid is not suggested. Vaginal entrapment of bathwater or other fluids of immersion has yet to be considered in the literature as a condition that may masquerade as urinary incontinence.

We present 2 cases of females presenting with what could be considered urinary leakage, but is actually secondary to vaginal entrapment of bathwater. One case is a premenopausal adult and the other is a prepubertal child.

\section{Methods}

Institutional Research Board approval was obtained for the retrospective and prospective assessment of these cases.
Data on the adult was obtained by direct interview and by performing the following 6 prospective studies: (1) measurement of volume of entrapped bathwater ; (2) determination of frequency of bathwater entrapment occurring; (3) determination of time interval in which fluid will spontaneously leak after a bath, if not evacuated; (4) tampon study to determine if an intra-vaginal tampon becomes wet during bathing; (5) confirmation that fluid from the vagina is bathwater (and not urine), using a $\mathrm{pH}$ and colour test. The evacuated vaginal fluid, concurrent bathwater and post bath voided urine were all assessed with visual examination and Chemstrip 7 by a non-blinded examiner the day after collection. A dye test was also performed. Red food colouring was added to the bathwater. Vaginal fluid was squeezed from a tampon and then visually compared to both concurrent bathwater and clean tap water by a blinded examiner the day after collection. (6) Genital examination by a primary care physician. Data was gathered on the child through a clinic chart review and a phone interview with the mother.

\section{Literature search}

A literature search was performed using PubMed, Embase and a Conference Papers Index. Search terms included "vagina AND bathwater," "vagina AND pool water," "vagina AND swimming," "vaginal entrapment," "unconscious incontinence," and "extra-urethral incontinence." Articles were read if the titles and abstracts were pertinent. The titles and abstracts of related articles and relevant referenced articles were also reviewed. Recent review articles on the diagnosis and management of female incontinence were reviewed, as were major textbooks in the specialties of urology and obstetrics \& gynecology (O\&G). As bathwater entrapment is related to the phenomena of urine entrapment from urethro-vaginal reflux, a search on this condition was also undertaken. 


\section{Results}

\section{Adult}

\section{Demographics/gynecological and urological history}

The adult patient is a 39-year-old G2P2 premenopausal woman, with history of Cesarean section and vaginal delivery with uncomplicated episiotomy. She uses an intrauterine device (IUD) and is sexually active. She has a remote history of recurrent cystitis and experiences mild stress urinary incontinence.

\section{Symptoms}

This woman has a long-standing history of post-bath dribbling. She cannot remember when it was first noted. She recalls initially experiencing dribbling which could soak through her underwear, causing her to change her clothing. It does not occur after swimming or hot-tubbing.

\section{Diagnosis}

She noted the temporal association of the dribbling occurring shortly after a bath and concluded that it represented bathwater in her vagina. Therefore, to avoid having to change her clothes or underwear after taking a bath, she made a habit of evacuating any entrapped bathwater from her vagina prior to stepping out of the tub. This is done by inserting a digit in the vagina and pulling laterally. The author became aware of her condition through casual disclosure, not through medical referral.

\section{Prospectively Performed Investigations}

1. Measurement of volume of entrapped bathwater: On 2 occasions, fluid was collected for measurement by holding a measuring cup under the introitus during evacuation. Volumes were $24 \mathrm{~mL}$ and $29 \mathrm{~mL}$.

2. Determination of frequency of bathwater entrapment occurring: Fluid is evacuated less than $50 \%$ of the time. She could not identify any variables that would predict entrapment.

3. Determination of time interval in which fluid will dribble after a bath if not evacuated: On the 1 occasion when she purposely elected not to evacuate, after 15 minutes the crotch of her underwear was soaked and her pants had a spot of wetness, so she changed her clothes. After another 10 minutes (for a total 25 minutes), her underwear was wet again. Before changing her underwear again, she digitally evacuated a minimal amount of remaining fluid, suggesting that at least 1 more episode of dribbling would have occurred beyond 25 minutes.

4. Tampon study to determine if a tampon becomes wet during bathing: On 3 occasions a tampon was placed intra-vaginally prior to the bath. Each time, the tampon was soaked with fluid early into the bath. On the last 2 occasions, the cotton string of the tampon was removed to ensure that bathwater was not wicking up the string.

5. Confirmation that fluid from the vagina is bathwater: A $\mathrm{pH}$ and colour test were performed (Table 1). A dye test was also done. Three attempts were made to collect entrapped fluid following a bath where the bathwater had been dyed with red food colouring. Unfortunately, fluid entrapment did not occur with any of these baths. Therefore, given that fluid entry into the vagina was predictable with use of a tampon, following the last attempt an intra-vaginal tampon, with the string cut, was inserted. Fluid squeezed from the tampon was then used for visual assessment (Table 1).

6. Genital exam by a primary care physician: An internal exam revealed nabothian cysts on the cervix and IUD strings at the cervical os. No other abnormalities detected. No abnormalities were detected on external examination.

\section{Child}

\section{Demographics/gynecological and urological history}

This child is an 8-year-old healthy pre-pubertal girl with no history of urinary tract infections or genital surgery.

\section{Symptoms}

This girl experienced an 8-month history of a minimal amount $(1 \mathrm{~cm}$ in diameter) of wetness on her underwear almost every morning. Occasionally, she noticed it before bed. It had the consistency and colour of a clear liquid with no notable odour. This small amount of wetness was quite bothersome. She was very sensitive to it, causing her to frequently wipe herself; a box of tissue was kept by the bed for this purpose. The symptoms were bothersome enough

Table 1. Confirmation that fluid from vagina is bathwater

\begin{tabular}{lccccc}
\hline \multicolumn{1}{c}{ Investigations } & & Voided urine & Evacuated/tampon fluid & Bathwater & Clean water \\
\hline $\begin{array}{l}\text { 5a) Regular } \\
\text { bathwater }\end{array}$ & $\mathrm{pH}$ & 5 & 7.5 & 7 & - \\
\hline 5b) Dyed bathwater & Colour & Clear yellow & Cloudy white & Clear colourless & Pink \\
\hline
\end{tabular}


to present to her primary care physician, who referred her to a pediatric urologist for the complaint of "constantly wetting herself."

\section{Investigations}

Neurological exam was normal. Genital exam revealed Tanner Stage I with no labial adhesions. Laboratory tests included urinalysis, urine culture and vaginal swab. Results were negative, negative and "mixed flora, no yeast," respectively. The voiding diary showed normal voiding frequency and functional bladder capacity. Times of wet underwear were recorded in the diary.

\section{Diagnosis}

As her urologist was already aware of the adult case, the history included a question regarding bathing. It was noted that the child baths almost every evening. However, on a recent vacation, where she did not have access to a bathtub, she recalled not being bothered by the symptoms. Review of the voiding diary revealed that the mornings with wet underwear corresponded to when a bath was taken the previous evening. Vaginal entrapment of bathwater was suspected. To confirm this, the child was instructed to go 1 week with only showering, and 1 week with bathing, and to note if the wetting was only associated with bathing. Results confirmed the association. Following the diagnosis, the child elected to shower instead of bath. When she does have the occasional bath, she will be wet.

\section{Literature search}

There is very little in the literature regarding whether fluid enters the vagina during bathing or swimming. There were only 2 very outdated references..$^{2,3}$ One was a study from 1960 performed in an effort to confirm that bathing was safe during pregnancy and the postpartum period. In this study, bathwater did not come in contact with an intravaginal tampon in 10 such women. The author concluded that bathwater does not normally enter the vagina. ${ }^{2}$ The second reference was in answer to a question on the issue of whether swimming or bathing post-coitus might impact the effectiveness of a diaphragm, given the possibility of fluid flooding the vagina. The response, written in 1977, suggested that the author did feel such fluids were capable of entering the vagina. ${ }^{3}$ On discussion with a local gynecologist [Dr. Margaret Morris, Department Head Obstetrics \& Gynecology, University of Manitoba, oral communication, August 27, 2009], it appears that the general belief amongst the O\&G community is that upon immersion, fluids such as bathwater can enter the vagina; however, it is not thought to be of any significance, as it is not thought to cause problems or become entrapped. However, an online review of major O\&G textbooks, searching for key words of "swimming" or "bathwater," revealed no reference to the topics., ${ }^{4,5}$

There are no references in the literature to bathwater or swim water entering the prepubertal vagina.

Regarding vaginal entrapment of urine secondary to urethra-vaginal reflux, although this is a common cause of incontinence in girls, it is not thought to occur in adults. ${ }^{6}$ However, we were able to find 2 adult cases in the literature. In 1 case, failure to make the diagnosis resulted in numerous unnecessary surgeries. ${ }^{7,8}$

\section{Discussion}

We have shown that bathwater can become entrapped in the vagina, resulting in bothersome dribbling in females with normal anatomy. Regarding the child, we believe that the temporal association of her symptoms with bathing allows us to conclude that the fluid is bathwater. While it would be ideal to provide more objective evidence of this, we felt it would be unethical to subject a child to further investigations. However, in the adult case, we were able to confirm the evacuated fluid was bathwater, showing similarities in both $\mathrm{pH}$ and colour. It would have been preferable if the vaginal specimen collected when the bathwater was dyed had been by evacuation rather than squeezed from a tampon. However, it is unlikely that the vagina fills with bathwater when there is a tampon in place and with another unidentified fluid when no tampon is in place. It is unknown why the tampon was consistently soaked, while fluid could not be evacuated after every bath. One possibility is that the tampon changed the anatomy of her vagina and increased the likeliness of fluid entering the vagina. It is also possible that bathwater routinely enters her vagina, but unknown variables determine whether it will become entrapped.

As vaginal entrapment of bathwater has not been described in the literature until now, it likely is rare. Alternatively, perhaps women who experience this have been able to identify it and deal with it discretely without presenting for medical attention, as was the case with our adult. If it can occur in a bathtub, one could extrapolate that it may occur in a hot tub or with swimming. However, it is not uncommon to remain in a wet swimsuit following such immersion, or to take a shower afterward. Therefore, if dribbling were to occur during these times, it would be less noticeable.

\section{Conclusion}

The phenomenon of urine becoming entrapped in the vagina, in both children and adults, has previously been identified, although it is rarely considered in adults. We have shown that fluids of immersion can also become entrapped in the vagina. While this source of extra-urethral incontinence is 
likely uncommon, it is important for those who specialize in the diagnosis and management of urinary incontinence to be aware of its existence. It was the author's awareness of this condition in an adult that led to its diagnosis in a child.

In cases of dribbling incontinence in females, a few simple questions can rule out vaginal entrapment of fluids of immersion. If the diagnosis is suspected then a period without immersion can identify a temporal relationship. Following diagnosis, the management is behavioural, which would include avoidance of immersion or techniques of evacuation.

The differential diagnosis of dribbling incontinence in females of any age should include the extra-urethral cause of entrapped fluids of immersion. Although this condition may be rare, it is simple to rule out on history based on its temporal association with fluid immersion. Once diagnosed, management is behavioural.

*Department of Urology, Winnipeg Children's Hospital, Assistant Professor, University of Manitoba, Winnipeg MB; 'Resident, University of Manitoba, Winnipeg, MB

Competing interests: None declared.

This paper has been peer-reviewed.

\section{References}

1. Abrams $\mathrm{P}$, Cardozo $\mathrm{L}$, Fall $M$, et al. The standardization of terminology in lower urinary tract function; report from the standardization sub-committee of the international continence society. Urology 2003;61:37-49.

2. Siegel P. Does bath water enter the vagina? Obstet Gynecol 1960;15:660-1.

3. Bernstein GS. Is effectiveness of diaphragm compromised by postcoital swimming or bathing? JAMA 1977;237:270

4. Gabbe SG, Niebyl JR, Simpson JL, editors. Obstetrics: Normal and Problem Preganancies. $5^{\text {th }}$ ed. Philadelphia: Churchill Livingston Elsevier; 2007.

5. Katz VL, Lentz G, Lobo RA, Gershenson. Comprehensive Gynecology. 5th edition. Philadelphia: Mosby Elsevier; 2007.

6. Mattsson $S$, Gladh $G$. Urethrovaginal reflux - a common cause of daytime incontinence in girls. Pediatr 2003;111:136-9.

7. Haouas N, Cariou G. Le reflux urétro-vaginal permictionnel: à propos d'un cas. J Gynecol Obstet Biol Reprod (Paris) 2006;35:621-3.

8. Nagi JB, Robinson D, Cardozo L, Anders K. Anatomical entrapment-an unusual cause of "urinary incontinence." J Obstet Gynaecol 2004;24:709-18.

Correspondence: Dr. Karen Psooy, Assistant Professor, University of Manitoba, AE301-840 Sherbrook St., Winnipeg, MB R3A 1S1; kpsooy@hsc.mb.ca 